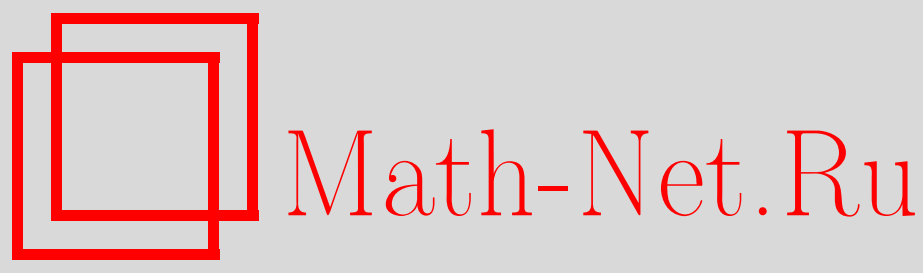

А. С. Рыбаков, Натуральные решения систем линейных уравнений и многочлены малого веса, делящиеся на (1$x)^{r}$, Дискрет. матем., 2014, том 26, выпуск 2, 131-142

DOI: https://doi.org/10.4213/dm1284

Использование Общероссийского математического портала Math-Net.Ru подразумевает, что вы прочитали и согласны с пользовательским соглашением http://www . mathnet.ru/rus/agreement

Параметры загрузки:

IP : 54.174 .149 .18

26 апреля 2023 г., 08:27:55

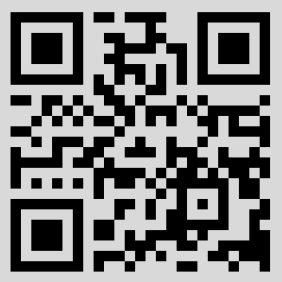




\title{
Натуральные решения систем линейных уравнений и многочлены малого веса, делящиеся на $(1-x)^{r}$
}

\author{
() 2014 г. А.С. Рыбаков*
}

\begin{abstract}
Получена верхняя оценка нормы одного из решений в натуральных числах произвольной системы линейных однородных уравнений с целыми коэффициентами, имеющей непустое множество натуральных решений. С помощью этой оценки установлена верхняя оценка нормы вектора, компоненты которого являются степенями двучленов, произведение которых даёт разностный многочлен минимально возможного веса. Последний результат имеет применения в теории антенных решёток с управляемыми диаграммами направленности.
\end{abstract}

Ключевые слова: натуральные решения систем линейных уравнений, многочлены малого веса, антенные решётки.

\section{1. Введение}

В данной работе получены оценки размеров некоторых решений в натуральных числах произвольных систем линейных однородных уравнений с целыми коэффициентами, имеющих непустое множество натуральных решений. Как будет показано ниже, такие оценки применимы при построении многочленов $G(x) \in \mathbb{Z}[x]$ с малыми коэффициентами, для которых точка $x=1$ является корнем большой кратности.

Многочлены с малыми коэффициентами, делящиеся на $(1-x)^{r}$, широко изучаются в зарубежной литературе, см., в частности, работы [6], [7], [8], [9], [10], [12] и другие. На практике такие многочлены применяются при проектировании линейных антенных решёток с полосно-заграждающими фильтрами (см. [9], [10], [13], [14]).

Отметим, что задача получения оценок целочисленных решений систем линейных однородных уравнений с целыми коэффициентами решается достаточно просто с помощью принципа Дирихле. Известен следующий результат.

Теорема I [5; гл. 1, §6.1, теор.6.1]. Пусть $m, n \in \mathbb{N}, m<n, a_{i, j} \in \mathbb{Z}, i=1, \ldots, m$, $j=1, \ldots, n$,

$$
A_{i} \in \mathbb{N}, \quad A_{i} \geqslant \sum_{j=1}^{n}\left|a_{i, j}\right|, \quad i=1, \ldots, m .
$$

Тогда система уравнений

$$
\sum_{j=1}^{n} a_{i, j} x_{j}=0, \quad i=1, \ldots, m
$$

имеет нетривиальное челое решение $x_{1,0}, \ldots, x_{n, 0}$, удовлетворяющее условию

* Место работы: Лаборатория ТВП, e-mail: ууу0х3@mail.ru 


$$
\max _{j=1, \ldots, n}\left|x_{j, 0}\right| \leqslant\left(A_{1} \cdot \ldots \cdot A_{m}\right)^{1 /(n-m)} .
$$

Далее будем через $\left|\bar{x}_{0}\right|_{2}$ обозначать евклидову длину вектора $\bar{x}_{0}$.

Первый основной результат настоящей работы аналогичен теореме I, но касается не целочисленных, а натуралъных решений линейных систем уравнений и формулируется следующим образом.

Теорема 1. Пусть $m, n \in \mathbb{N}, a_{i, j} \in \mathbb{Z}, i=1, \ldots, m, j=1, \ldots, n$ и а-максимум модулей чисел $a_{i, j}$. Предположим, что система линейных однородных уравнений (1) имеет решение с натуральными компонентами. Тогда эта система имеет решение $\bar{x}_{0}=\left(x_{1,0}, \ldots, x_{n, 0}\right)$ с натуральными компонентами $x_{j, 0}$, удовлетворяющее неравенству

$$
\left|\bar{x}_{0}\right|_{2} \leqslant 4 n^{11 / 4} \cdot\left(\frac{n+1}{\sqrt{8 e}}\right)^{(n+1) / 2} \cdot\left(\frac{n+1}{2} \cdot e^{1 / \ln (n+1)} a^{2}\right)^{(n+1)^{2} / 8},
$$

где $\left|\bar{x}_{0}\right|_{2}-$ длина вектора $\bar{x}_{0}$.

С помощью этой теоремы доказывается следующее утверждение, являющееся другим основным результатом нашей работы.

Теорема 2. При любом $r$ множество векторов $\bar{h}=\left(h_{1}, \ldots, h_{r}\right)$, на которьх достигается минимум веса (суммы модулей коэффициентов) многочлена

$$
G(x)=\prod_{j=1}^{r}\left(1-x^{h_{j}}\right),
$$

содержит вектор $\bar{h}_{0}$, длина которого удовлетворяет верхней оценке

$$
\left|\bar{h}_{0}\right|_{2} \leqslant 4 r^{11 / 4} \cdot\left(\frac{r+1}{\sqrt{8 e}}\right)^{(r+1) / 2} \cdot e^{(r+1)^{2} /(8 \ln (r+1))} \cdot\left(\frac{r+1}{2}\right)^{(r+1)^{2} / 8} .
$$

Теоремы 1 и 2, имеющие вполне самостоятельный интерес, применимы к задаче построения многочленов с малыми коэффициентами, делящихся на $(1-x)^{r}$. Для построения хорошего полосно-заграждающего фильтра (см. [9], [10]) надо, чтобы коэффициенты такого многочлена были очень малы (в идеале - $0, \pm 1$ ), а степень не слишком велика (скажем, $O\left(r^{2}\right)$ при больших $r$ ). Многочлены $G(x) \in \mathbb{Z}[x]$, делящиеся на $(1-x)^{r}$, будем далее для краткости называть $r$-многочленами.

Легко построить $r$-многочлен с коэффициентами $0, \pm 1$, степени порядка $2^{r}$. Например, можно взять многочлен $G(x)=\prod_{j=1}^{r}\left(1-x^{2^{j}}\right)$. Понятно, что такие многочлены представляют практический интерес лишь при достаточно малых значениях параметра $r$.

Пользуясь принципом Дирихле, легко показать (см. [12] и [10]), что при любом $r$ существует $r$-многочлен степени $O\left(r^{2} \ln r\right)$ с коэффициентами $0, \pm 1$. Более точно, для степени $D$ такого многочлена можно получить оценку

$$
D \leqslant r^{2} \ln r \cdot \frac{1}{2 \ln 2}+C r^{2} \ln \ln r,
$$

где $C>0$ - достаточно большая константа. Следовательно, трудоёмкость полного перебора, с помощью которого можно найти такой многочлен, составляет не более

$$
3^{r^{2} \ln r /(2 \ln 2)+O\left(r^{2} \ln \ln r\right)}
$$


С другой стороны, наименьшая возможная степень ненулевого $r$-многочлена с коэффициентами $0, \pm 1$ ограничена снизу величиной $c r^{2}$, где $c>0$ - константа (см. [7]). Это показывает, что $r$-многочлен с коэффициентами $0, \pm 1$, обеспечиваемый принципом Дирихле, имеет почти минимально возможную степень. Однако найти такой многочлен практически возможно лишь при очень малых значениях параметра $r$.

Автор провел множество компьютерных экспериментов, в которых генерировались $r$-многочлены с малыми коэффициентами (не обязательно только $0, \pm 1$ ) путём поиска ненулевых коротких векторов решёток

$$
E(d, r)=\left\{\left(f_{0}, \ldots, f_{d}\right):(1-x)^{r} \mid f(x)=\sum_{i=0}^{d} f_{i} x^{i} \in \mathbb{Z}[x]\right\}
$$

с величиной $d$ порядка $O\left(r^{2}\right)$. Для нахождения коротких векторов использовался известный алгоритм BKZ редукции базисов решёток [15], реализованный в теоретико-числовом пакете NTL [16], имеющемся в свободном доступе в сети Интернет.

Для сравнительной оценки генерируемых $r$-многочленов, которые могут отличаться друг от друга по двум критически важным для приложений параметрам, a именно, по степени и по максимуму модулей коэффициентов, была выбрана функция веса (сумма модулей коэффициентов). Многочлены малого веса имели во всех случаях относительно низкую степень и хорошо сбалансированный набор коэффициентов, что показывает, что данная целевая функция является вполне адекватной.

Эксперименты продемонстрировали весьма интересный феномен: во всех случаях $r$-многочлен $f(x)=\sum_{i=0}^{d} f_{i} x^{i}$, соответствующий первому вектору BKZ-редуцированного базиса решётки $E(d, r)$, совпадал с некоторым многочленом вида (2). Автор не обнаружил ни одного контрпримера, который опровергал бы эту закономерность.

Заметим, что из определения BKZ-редуцированного базиса следует, что длина первого вектора такого базиса близка к длине кратчайшего ненулевого вектора решётки [15]. Поэтому, ввиду сбалансированности, первый вектор такого базиса имеет и близкий к минимуму вес.

Поскольку многочлены вида (2) (такие многочлены называются разностными) являются хорошим источником получения $r$-многочленов малого веса, естественно сосредоточить на них отдельное внимание. Назовём разностный многочлен (2) $r$-оптимальным, если он имеет наименьший вес среди всех разностных многочленов с тем же значением $r$.

Заметим, что, вообще говоря, неясно, как искать $r$-оптимальный разностный многочлен, поскольку множество векторов $\left(h_{1}, \ldots, h_{r}\right)$, среди которых надо найти вектор, минимизирующий вес многочлена (2), счётно. Автору не известны результаты, в которых была бы приведена верхняя оценка трудоёмкости построения $r$-оптимальных разностных многочленов.

Теорема 2, дающая верхнюю оценку длины вектора $\left(h_{1}, \ldots, h_{r}\right)$, минимизирующего вес многочлена (2), является первым эффективным результатом в этом направлении. Теорема показывает, что трудоёмкость нахождения такого набора хотя и велика, но ограничена сверху величиной, зависящей только от $r$. Отметим, что при больших $r$ правая часть формулы (3) имеет вид $r^{r^{2} / 8+O\left(r^{2} / \ln r\right)}$, а правая часть

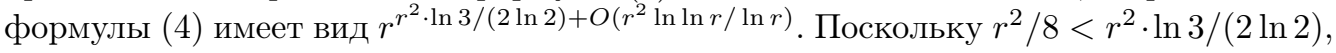
можно ожидать, что при достаточно больших значениях $r$ трудоёмкость нахождения $r$-оптимального разностного многочлена методом полного перебора будет меньше, 
чем трудоёмкость нахождения $r$-многочлена минимально возможной степени с коэффициентами $0, \pm 1$ аналогичным методом. Экспериментов, подтверждающих или опровергающих это предположение, пока не проводилось.

\section{2. Фундаментальные системы ограниченных цело- численных решений систем линейных однородных уравнений}

Следующая лемма является некоторым аналогом сформулированной выше теоремы I, но даёт верхнюю оценку не для одного нетривиального решения системы целочисленных линейных однородных уравнений, а для базиса таких решений. Эта лемма показывает, что среди всевозможных базисов можно выбрать базис, компоненты векторов которого эффективно ограничены.

Лемма 1. Пусть $m, n \in \mathbb{N}, a_{i, j} \in \mathbb{Z}, i=1, \ldots, m, j=1, \ldots, n$. Пусть $k$ - строчный ранг матрицы $A=\left(a_{i, j}\right), 1 \leqslant k<n$. Тогда решетка в $\mathbb{Z}^{n}$, являющаяся множеством целочисленных решений системы линейных однородных уравнений

$$
\sum_{j=1}^{n} a_{i, j} x_{j}=0, \quad i=1, \ldots, m,
$$

имеет базис $\bar{b}_{1}, \ldots, \bar{b}_{n-k}$, обладающий следующим свойством. При любом $i$ имеет место неравенство $\left|\bar{b}_{i}\right|_{2} \leqslant \Delta \max (\sqrt{n}, \sqrt{n i / 4})$, где $\Delta-$ максимальный из модулей $k$-миноров матрицы $A$.

Доказательство. Без ограничения общности можно считать, что строки

$$
\left(a_{i, 1}, \ldots, a_{i, n}\right), \quad i=1, \ldots, k
$$

матрицы $A$ линейно независимы. Пусть $\mathcal{L}$ - решетка, определяемая множеством целочисленных решений системы уравнений $(5)$ и $r=\operatorname{rk}(\mathcal{L})$. По известной теореме линейной алгебры имеет место равенство $k=n-r$. Покажем сначала, что в $\mathcal{L}$ имеется линейно независимая система векторов $\bar{v}_{1}, \ldots, \bar{v}_{r}$, координаты которых ограничены по модулю величиной $\Delta$.

Пусть $\bar{e}_{i} \in \mathbb{R}^{n}$ - вектор, все координаты которого равны 0 , за исключением $i$-й координаты, равной 1. По известной теореме линейной алгебры матрицу

$$
A_{0}=\left(a_{i, j}: i=1, \ldots, k ; j=1, \ldots, n\right)
$$

можно дополнить строками $\bar{e}_{i}, i=1, \ldots, n$ до невырожденной квадратной матрицы $A_{1}$. Без ограничения общности можно считать, что система векторов, дополняющая матрицу $A_{0}$ до матрицы

$$
A_{1}=\left(a_{i, j}: i=1, \ldots, n ; j=1, \ldots, n\right),
$$

совпадает с системой векторов $\bar{e}_{k+1}, \ldots, \bar{e}_{n}$. Рассмотрим систему векторов $\bar{v}_{i}, i=$ $1, \ldots, r$, удовлетворяющих равенствам

$$
A_{1} \bar{v}_{i}=\Delta_{0} \bar{e}_{k+i}, \quad i=1, \ldots, r
$$

где $\Delta_{0}$ - минор матрицы $A$, состоящий из строк с номерами $1, \ldots, k$ и столбцов с номерами $1, \ldots, k$. Из построения матрицы $A_{1}$ вытекает, что число $\Delta_{0}$ является 
определителем этой матрицы и $\Delta_{0} \neq 0$. Пусть $A_{1}^{*}$ - матрица, сопряженная к матрице $A_{1}$. По определению матрица $A_{1}^{*}$ имеет вид $A_{1}^{*}=\left(A_{1}^{(i, j)}\right)^{t}$, где $A_{1}^{(i, j)}$ - алгебраическое дополнение к элементу $a_{i, j}$ матрицы $A_{1}$ и $^{t}$ - операция транспонирования. Домножая равенство (6) слева на $A_{1}^{*}$ и пользуясь известной теоремой теории определителей, получим

$$
\bar{v}_{i}=A_{1}^{*} \bar{e}_{k+i} \quad \text { для } i=1, \ldots, r .
$$

Из формулы (6) следует, что векторы $\bar{v}_{1}, \ldots, \bar{v}_{r}$ принадлежат решетке $\mathcal{L}$ и линейно независимы. Поскольку их $r$ штук, они образуют максимальную линейно независимую систему векторов решетки.

Из формулы (7) следует, что компонентами вектора $\bar{v}_{i}$ являются алгебраические дополнения $A_{1}^{(k+i, j)}, j=1, \ldots, n$. Легко заметить, что число $\left|A_{1}^{(k+i, j)}\right|$ равно нулю при $j>k, j \neq k+i$, величине $\Delta_{0}$ при $j=k+i$ и модулю $k$-минора матрицы $A_{0}$, составленного из строк $1, \ldots, k$ и столбцов $1, \ldots, j-1, j+1, \ldots, k, k+i$ при $j \leqslant k$. Таким образом, компоненты всех векторов $\bar{v}_{i}$ ограничены по модулю величиной $\Delta$, и наше утверждение доказано.

Теперь покажем, что в решетке $\mathcal{L}$ имеется базис $\bar{b}_{1}, \ldots, \bar{b}_{r}$, обладающий указанным в лемме 1 свойством.

Рассмотрим множество $S_{1}$ целых чисел, являющихся $(k+1)$-ми координатами векторов $A_{1} \bar{v}$, где $\bar{v}$ - векторы решетки $\mathcal{L}$. Легко видеть, что множество $S_{1}$ является идеалом кольца $\mathbb{Z}$. Поскольку в $\mathbb{Z}$ все идеалы - главные, $S_{1}=\left(d_{1}\right)$ для некоторого целого числа $d_{1}$. Число $d_{1}$ не равно нулю, поскольку ввиду равенств $(6)$ число $\Delta_{0}$ принадлежит $S_{1}$. Не ограничивая общности, можно считать, что $d_{1}>0$. Пусть $\bar{v}_{1,0}$ - вектор решетки, для которого $(k+1)$-я координата вектора $A_{1} \bar{v}_{1,0}$ равна $d_{1}$. Вычитая из вектора $\bar{v}_{1,0}$ подходящую линейную комбинацию векторов $\bar{v}_{2}, \ldots, \bar{v}_{r}$, можно считать, что остальные ненулевые координаты вектора $A_{1} \bar{v}_{1,0}$ по модулю не превосходят $\left|\Delta_{0}\right| / 2$. Таким образом, можно записать, что

$$
\begin{gathered}
A_{1} \bar{v}_{1,0}=\bar{u}_{1}, \quad \bar{u}_{1}=\left(u_{1,0}, \ldots, u_{1, n}\right)^{t}, \\
u_{1, i}=0 \text { при } i \leqslant k, \quad u_{1, k+1}=d_{1}, \quad\left|u_{1, i}\right| \leqslant\left|\Delta_{0}\right| / 2 \text { при } i>k+1 .
\end{gathered}
$$

Из (8) следует, что

$$
\bar{v}_{1,0}=\frac{1}{\Delta_{0}} A_{1}^{*} \bar{u}_{1}=\frac{1}{\Delta_{0}} \sum_{i=1}^{n} u_{1, i} A_{1}^{*} \bar{e}_{i}=\frac{1}{\Delta_{0}} \sum_{i>k} u_{1, i} \bar{v}_{i-k} .
$$

Поскольку длины векторов $\bar{v}_{i-k}$ по доказанному не превосходят величины $\sqrt{n} \Delta$, из последних равенств и (8) в силу известного неравенства Коши - Буняковского Шварца имеем

$$
\left|\bar{v}_{1,0}\right|_{2} \leqslant \sqrt{d_{1}^{2} / \Delta_{0}^{2}+(r-1) / 4} \cdot \sqrt{n} \Delta .
$$

Рассмотрим множество $S_{2}$ целых чисел, являющихся $(k+2)$-ми координатами векторов $A_{1} \bar{v}$, где $\bar{v}$ - векторы решетки $\mathcal{L}$, для которых $(k+1)$-я координата вектора $A_{1} \bar{v}$ равна нулю. Легко видеть, что множество $S_{2}$ является идеалом кольца $\mathbb{Z}$. Поскольку в $\mathbb{Z}$ все идеалы - главные, то $S_{2}=\left(d_{2}\right)$ для некоторого целого числа $d_{2}$. Число $d_{2}$ не равно нулю, поскольку ввиду равенств (6) число $\Delta_{0}$ принадлежит $S_{2}$. Не ограничивая общности, можно считать, что $d_{2}>0$. Пусть $\bar{v}_{2,0}-$ вектор решетки, для которого $(k+1)$-я координата вектора $A_{1} \bar{v}_{2,0}$ равна нулю, а $(k+2)$-я координата вектора $A_{1} \bar{v}_{2,0}$ равна $d_{2}$. Вычитая из вектора $\bar{v}_{2,0}$ подходящую линейную комбинацию векторов $\bar{v}_{3}, \ldots, \bar{v}_{r}$, можно считать, что остальные ненулевые координаты вектора $A_{1} \bar{v}_{2,0}$ по модулю не превосходят $\left|\Delta_{0}\right| / 2$. Таким образом, 


$$
\begin{aligned}
& A_{1} \bar{v}_{2,0}=\bar{u}_{2}, \quad u_{2}=\left(u_{2,0}, \ldots, u_{2, n}\right)^{t}, \\
& u_{2, i}=0 \text { при } i \leqslant k+1, \quad u_{2, k+2}=d_{2}, \quad\left|u_{2, i}\right| \leqslant\left|\Delta_{0}\right| / 2 \text { при } i>k+2 .
\end{aligned}
$$

Из (9) следует, что

$$
\bar{v}_{2,0}=\frac{1}{\Delta_{0}} A_{1}^{*} \bar{u}_{2}=\frac{1}{\Delta_{0}} \sum_{i=1}^{n} u_{2, i} A_{1}^{*} \bar{e}_{i}=\frac{1}{\Delta_{0}} \sum_{i>k+1} u_{2, i} \bar{v}_{i-k} .
$$

Отсюда и из (8) с помощью неравенства Коши - Буняковского - Шварца получим, что

$$
\left|\bar{v}_{2,0}\right|_{2} \leqslant \sqrt{d_{2}^{2} / \Delta_{0}^{2}+(r-2) / 4} \cdot \sqrt{n} \Delta .
$$

Продолжая аналогичным образом, можно построить систему векторов $\bar{v}_{1,0}, \ldots, \bar{v}_{r, 0}$ решетки и систему чисел $d_{1}, \ldots, d_{r}$, обладающих следующими свойствами.

(1) Векторы $\bar{u}_{i}=A_{1} \bar{v}_{i, 0}$ имеют вид $\bar{u}_{i}=\left(u_{i, 0}, \ldots, u_{i, n}\right)^{t}$, где $u_{i, j}=0$ при $j<k+i$, $u_{i, k+i}=d_{i}, d_{i}>0, d_{i} \mid \Delta_{0}$ и $\left|u_{i, j}\right| \leqslant\left|\Delta_{0}\right| / 2$ при $j>k+i$.

(2) Для всех чисел $i=1, \ldots, r$ длины векторов $\bar{v}_{i, 0}$ удовлетворяют неравенствам $\left|\bar{v}_{i, 0}\right|_{2} \leqslant \sqrt{d_{i}^{2} / \Delta_{0}^{2}+(r-i) / 4} \cdot \sqrt{n} \Delta$.

(3) Для произвольного вектора $\bar{v}$ решетки имеет место следующее утверждение. Пусть $\bar{w}=A_{1} \bar{v}=\left(w_{1}, \ldots, w_{n}\right)^{t}$ и пусть $j$ - минимальный индекс, для которого $w_{j} \neq 0$. Тогда $j>k$ и $d_{j-k} \mid w_{j}$.

Из свойств (1) и (3) легко вывести, что векторы $\bar{v}_{1,0}, \ldots, \bar{v}_{r, 0}$ образуют базис решетки $\mathcal{L}$. Действительно, пусть $\bar{g}_{1}-$ произвольный ненулевой вектор решетки. Покажем, что вектор $\bar{g}_{1}$ выражается через векторы $\bar{v}_{i, 0}, i=1, \ldots, r$, с целыми коэффициентами. Рассмотрим для этого вектор $\bar{h}_{1}=A_{1} \bar{g}_{1}=\left(h_{1,1}, \ldots, h_{1, n}\right)^{t}$. Пусть $j_{1}>k$ - минимальный из таких индексов $j$, что $h_{1, j} \neq 0$. Тогда $h_{1, j_{1}}=\lambda_{1} d_{j_{1}-k}$ для некоторого целого $\lambda_{1}$. Положим $\bar{g}_{2}=\bar{g}_{1}-\lambda_{1} \bar{v}_{j_{1}-k, 0}$ и $\bar{h}_{2}=A_{1} \bar{g}_{2}=\left(h_{2,1}, \ldots, h_{2, n}\right)^{t}$. Если вектор $\bar{g}_{2}$ равен нулю, то наше утверждение доказано. В противном случае вектор $\bar{h}_{2}$ также не равен нулю. Пусть $j_{2}>k$ - минимальный из таких индексов $j$, что $h_{2, j} \neq 0$. Тогда $h_{2, j_{2}}=\lambda_{2} d_{j_{2}-k}$ для некоторого целого $\lambda_{2}$. Положим $\bar{g}_{3}=\bar{g}_{2}-\lambda_{2} \bar{v}_{j_{2}-k, 0}$ и $\bar{h}_{3}=A_{1} \bar{g}_{3}=\left(h_{3,1}, \ldots, h_{3, n}\right)^{t}$. Продолжая аналогично, получим, что на некотором $t$-м шаге вектор

$$
\bar{g}_{t}=\bar{g}_{1}-\lambda_{1} \bar{v}_{j_{1}-k, 0}-\ldots-\lambda_{t-1} \bar{v}_{j_{t-1}-k, 0}
$$

будет равен нулю, и наше утверждение доказано.

Пусть $i_{1}, \ldots, i_{s}$ - это точно те индексы, для которых $d_{i}=\left|\Delta_{0}\right|$. Для остальных индексов имеем $d_{i}<\left|\Delta_{0}\right|$. Так как числа $d_{i}$ делят $\Delta_{0}$, то $d_{i} \leqslant\left|\Delta_{0}\right| / 2$ для $i \neq i_{1}, \ldots, i_{s}$. Если мы в базисе $\bar{v}_{1,0}, \ldots, \bar{v}_{r, 0}$ заменим векторы $\bar{v}_{i, 0}$ векторами $\bar{v}_{i}$ при $i=i_{1}, \ldots, i_{s}$, то по доказанному мы получим также базис решётки. Обозначим векторы этого базиса через $\bar{v}_{1,1}, \ldots, \bar{v}_{r, 1}$. Тогда для длин этих векторов справедливы следующие оценки:

$\left|\bar{v}_{i, 1}\right| \leqslant \sqrt{n} \Delta$ при $i=i_{1}, \ldots, i_{s} ;$

$\left|\bar{v}_{i, 1}\right| \leqslant \sqrt{n(r-i+1) / 4} \Delta$ при $i \neq i_{1}, \ldots, i_{s}$.

Положим $\bar{b}_{i}=\bar{v}_{r-i+1,1}$ для всех $i$. Тогда векторы $\bar{b}_{1}, \ldots, \bar{b}_{r}$ образуют базис, обладающий указанными в формулировке леммы свойством. Лемма доказана.

Замечание 1. В начале доказательства леммы 1 было показано, что решётка $\mathcal{L}$ имеет $r$ линейно независимых векторов $v_{1}, \ldots, v_{r}$, длины которых не превосходят величины $\sqrt{n} \Delta$. Отсюда непосредственно следует, что $r$-й последовательный минимум Минковского [1] $\lambda_{r}=\lambda_{r}(\mathcal{L})$ решётки $\mathcal{L}$ не превосходит $\sqrt{n} \Delta$. Согласно известной оценке для длин векторов базиса Коркина - Золотарёва решётки [15; теорема 
$2.2]$ в $\mathcal{L}$ существует базис $\bar{b}_{1}^{\prime}, \ldots, \bar{b}_{r}^{\prime}$, длины векторов которого удовлетворяют оценке $\left|\bar{b}_{i}^{\prime}\right| \leqslant \sqrt{(i+3) / 4} \lambda_{r}$. Отсюда сразу вытекает, что $\left|\bar{b}_{i}^{\prime}\right| \leqslant \sqrt{n(i+3) / 4} \Delta$. Сравнивая это с оценками длин векторов базиса $b_{1}, \ldots, b_{n}$, видим, что последний редуцирован чуть лучше, чем базис Коркина - Золотарёва.

Следствие 1. Пусть выполнены условия леммы 1, а-максимум модулей элементов матрицы $A$. Тогда решетка в $\mathbb{Z}^{n}$, определяемая системой линейных однородных уравнений (5), имеет базис $\bar{b}_{1}, \ldots, \bar{b}_{n-k}$, обладающий следующим свойством: при любом $i$ имеет место неравенство $\left|\bar{b}_{i}\right|_{2} \leqslant k^{k / 2} a^{k} \max (\sqrt{n}, \sqrt{n i / 4})$.

Доказательство. В силу неравенства Адамара модули всех $k$-миноров матрицы $A$ ограничены сверху величиной $k^{k / 2} a^{k}$. Применяя это к оценкам леммы 1 , получим требуемое.

\section{3. Ограниченные натуральные решения систем ли- нейных однородных уравнений}

В следующей лемме даётся необходимая для дальнейшего исходная верхняя оценка для одного из натуральных решений системы целочисленных линейных однородных уравнений при условии, что рассматриваемая система имеет непустое множество натуральных решений.

Лемма 2. Пусть $m, n \in \mathbb{N}, a_{i, j} \in \mathbb{Z}, i=1, \ldots, m, j=1, \ldots, n$. Пусть $k$ - строчный ранг матрищы $A=\left(a_{i, j}\right), 1 \leqslant k<n$. Предположим, что система линейных однородных уравнений (5) имеет решение с натуральными компонентами. Тогда эта система имеет решение $\bar{x}=\left(x_{1,0}, \ldots, x_{n, 0}\right)$ с натуральными компонентами $x_{j, 0}$, удовлетворяющее неравенству

$$
|\bar{x}|_{2} \leqslant 4(r+1)^{9 / 4} \sqrt{n} \cdot\left(\frac{n(r+1)}{4 e}\right)^{(r+1) / 2} \cdot \Delta^{r+1},
$$

где $r=n-k u \Delta-$ максимальный из модулей $k$-миноров матрицы $A$.

Доказательство. По лемме 1 решетка в $\mathbb{Z}^{n}$, определяемая системой уравнений (5), имеет базис $\bar{b}_{1}, \ldots, \bar{b}_{r}$, длины векторов которого удовлетворяют неравенствам

$$
\left|\bar{b}_{i}\right|_{2} \leqslant \max (n, n i / 4)^{1 / 2} \cdot \Delta, \quad i=1, \ldots, r .
$$

Пусть $\bar{b}_{i}=\left(b_{i, 1}, \ldots, b_{i, n}\right)^{t}, i=1, \ldots, r$. Рассмотрим систему линейных неравенств

$$
y_{1} b_{1, j}+\ldots+y_{r} b_{r, j} \geqslant 1, \quad j=1, \ldots, n,
$$

относительно неизвестных целых чисел $y_{1}, \ldots, y_{r}$. Из условия леммы 2 следует, что эта система имеет целочисленное решение.

Сначала рассмотрим случай, когда $r=1$. В этом случае из того, что система (12) имеет целочисленное решение, вытекает, что либо все компоненты вектора $\bar{b}_{1}$ положительны, либо все эти компоненты отрицательны. Не ограничивая общности, можно считать, что все компоненты вектора $\bar{b}_{1}$ положительны. Покажем, что тогда вектор $\bar{b}_{1}$ и будет искомым натуральным решением системы (5), т.е. имеет место оценка (10). Поскольку вектор $\bar{b}_{1}$ удовлетворяет оценке (11), достаточно показать, что

$$
\sqrt{n} \Delta \leqslant 2^{17 / 4} \sqrt{n} \cdot \frac{n}{2 e} \cdot \Delta^{2}
$$


Последнее неравенство очевидно, и этим случай $r=1$ полностью исчерпан.

Далее при доказательстве леммы 2 считаем, что $r \geqslant 2$.

Пусть $B$ - матрица системы $(12)$ и $\bar{e}=(1, \ldots, 1)^{t} \in \mathbb{Z}^{n}$. Из теоремы 17.1 главы 17 монографии [4] следует, что система (12) имеет целочисленное решение $y_{1,0}, \ldots, y_{r, 0}$, компоненты которого удовлетворяют неравенствам $\left|y_{i, 0}\right| \leqslant(r+1) \Delta_{0}$, где $\Delta_{0}$ - максимальный из модулей миноров матрицы $[B \bar{e}]$.

Действительно, согласно формуле (1), приведенной в формулировке этой теоремы, целочисленная оболочка многогранника $P=\left\{\bar{y} \in \mathbb{R}^{r}: B^{t} \bar{y} \geqslant \bar{e}\right\}$ представляется в виде суммы выпуклой оболочки векторов $\bar{x}_{i} \in \mathbb{Z}^{r}, i=1, \ldots, t$, компоненты которых по модулю не превосходят $(r+1) \Delta_{0}$, и конуса, порождённого векторами $\bar{x}_{j}^{\prime} \in \mathbb{Z}^{r}$, $j=1, \ldots, s$, компоненты которых удовлетворяют тому же условию.

По крайней мере одно из чисел $s, t$ отлично от нуля, поскольку многогранник $P$ содержит целую точку. Если $t \neq 0$, то точка $\bar{x}_{1}$ будет требуемым целочисленным решением, а если $t=0$, то таким решением будет точка $\bar{x}_{1}^{\prime}$, что и требовалось.

В силу неравенства Адамара и неравенств (11) имеет место оценка сверху

$$
\Delta_{0} \leqslant \sqrt{n} \cdot \prod_{i=1}^{r}(\Delta \max (\sqrt{n}, \sqrt{n i / 4}))
$$

Отсюда следует, что

$$
\Delta_{0} \leqslant \sqrt{n} \cdot(\sqrt{n} \Delta)^{r} \cdot\left(\frac{r !}{6}\right)^{1 / 2}\left(\frac{1}{4^{r-3}}\right)^{1 / 2} \quad \text { при } r \geqslant 3
$$

И

$$
\Delta_{0} \leqslant \sqrt{n} \cdot(\sqrt{n} \Delta)^{r} \quad \text { при } r=2 .
$$

Определим набор чисел $x_{1,0}, \ldots, x_{n, 0}$ из равенств

$$
x_{j, 0}=y_{1,0} b_{1, j}+\ldots+y_{r, 0} b_{r, j}, \quad j=1, \ldots, n .
$$

По своему определению набор $x_{1,0}, \ldots, x_{n, 0}$ является решением системы линейных однородных уравнений (5) и все компоненты этого набора - натуральные числа. Пользуясь известным неравенством Коши - Буняковского - Шварца, можно написать следующий ряд неравенств:

$$
x_{j, 0}^{2} \leqslant\left(\sum_{i=1}^{r} y_{i, 0}^{2}\right) \cdot\left(\sum_{i=1}^{r} b_{i, j}^{2}\right), \quad j=1, \ldots, n .
$$

Суммируя эти неравенства по $j=1, \ldots, n$ и применяя оценки $(11)$, получим, что

$$
\begin{aligned}
|\bar{x}|_{2}^{2} & =\sum_{j=1}^{n} x_{j, 0}^{2} \leqslant\left(r(r+1)^{2} \Delta_{0}^{2}\right) \cdot\left(\sum_{i=1}^{r} \sum_{j=1}^{n} b_{i, j}^{2}\right) \\
& \leqslant\left(r(r+1)^{2} \Delta_{0}^{2}\right) \cdot\left(\sum_{i=1}^{r} \Delta^{2} \max (n, n i / 4)\right) .
\end{aligned}
$$

Отсюда

$$
|\bar{x}|_{2}^{2} \leqslant\left(r(r+1)^{2} \Delta_{0}^{2}\right) \cdot n \Delta^{2}\left(\frac{r(r+1)}{8}+\frac{6}{4}\right) \quad \text { при } r \geqslant 3
$$

$$
|\bar{x}|_{2}^{2} \leqslant\left(r(r+1)^{2} \Delta_{0}^{2}\right) \cdot r n \Delta^{2} \quad \text { при } r=2 \text {. }
$$


Пользуясь оценками $(13)$ и $(14)$ для величины $\Delta_{0}$, теперь получим, что

$$
|\bar{x}|_{2} \leqslant(\sqrt{n} \Delta)^{r+1} \cdot \sqrt{r n}(r+1)\left(\frac{r !}{6}\right)^{1 / 2} \sqrt{\frac{r(r+1)}{8}+\frac{6}{4}} \cdot\left(\frac{1}{4^{r-3}}\right)^{1 / 2} \quad \text { при } r \geqslant 3
$$

И

$$
|\bar{x}|_{2} \leqslant(\sqrt{n} \Delta)^{r+1} \cdot r(r+1) \sqrt{n} \quad \text { при } r=2 .
$$

Рассмотрим отдельно случай $r=2$. Ввиду (18) для доказательства леммы в этом случае достаточно показать, что

$$
(\sqrt{n} \Delta)^{r+1} \cdot 6 \sqrt{n} \leqslant(\sqrt{n} \Delta)^{r+1} \cdot 4 \cdot 3^{9 / 4} \sqrt{n} \cdot\left(\frac{3}{4 e}\right)^{3 / 2} .
$$

Но последнее неравенство выполнено, поскольку $4 \cdot 3^{9 / 4} \cdot(3 /(4 e))^{3 / 2}=6.8664 \ldots$ Этим случай $r=2$ исчерпан полностью.

Далее при доказательстве леммы 2 считаем, что $r \geqslant 3$.

По формуле Стирлинга [2; п.21.4-2, формула (21.4-12)] при любом натуральном $m$ имеет место оценка $m !<(m / e)^{m} \sqrt{2 \pi m} \cdot e^{1 /(12 m)}$. Следовательно,

$$
(r+1) !<((r+1) / e)^{r+1} \sqrt{2 \pi(r+1)} \cdot e^{1 /(12(r+1))} .
$$

Подставляя это в (17), получим, что

$$
|\bar{x}|_{2} \leqslant\left(\frac{n(r+1)}{4 e}\right)^{(r+1) / 2} \Delta^{r+1} \cdot\left(\frac{\sqrt{2 \pi} \cdot n r(r+1)^{3 / 2} 4^{4}}{6}\right)^{1 / 2} \cdot\left(\frac{r(r+1)}{8}+\frac{6}{4}\right)^{1 / 2} \cdot e^{1 /(24(r+1))} .
$$

Полагая $\rho=r+1$, последнее можно переписать в виде

$$
|\bar{x}|_{2} \leqslant\left(\frac{n \rho}{4 e}\right)^{\rho / 2} \Delta^{\rho} \cdot\left(\frac{\sqrt{2 \pi} \cdot n(\rho-1) \rho^{3 / 2} 4^{4}}{6}\right)^{1 / 2} \cdot\left(\frac{(\rho-1) \rho}{8}+\frac{6}{4}\right)^{1 / 2} \cdot e^{1 /(24 \rho)} .
$$

Пусть $f(\rho)=\left((\rho-1)^{2} \rho+12(\rho-1)\right) e^{1 /(12 \rho)} / \rho^{3}$. Тогда (19) переписывается в виде

$$
|\bar{x}|_{2} \leqslant\left(\frac{n \rho}{4 e}\right)^{\rho / 2} \Delta^{\rho} \cdot\left(\frac{\sqrt{2 \pi} \cdot 4^{4} n \rho^{9 / 2}}{48}\right)^{1 / 2} \cdot(f(\rho))^{1 / 2} .
$$

Путём непосредственной проверки нетрудно убедиться, что при $\rho \geqslant 4$ функция $\rho \rightarrow$ $f(\rho)$ сначала монотонно убывает от $f(4)=1.1486 \ldots$ до $f(11.9502 \ldots)=0.92305 \ldots$, а затем монотонно растёт до 1. Следовательно,

$$
|\bar{x}|_{2} \leqslant\left(\frac{n \rho}{4 e}\right)^{\rho / 2} \Delta^{\rho} \cdot\left(\frac{\sqrt{2 \pi} \cdot 4^{4} n \rho^{9 / 2}}{48}\right)^{1 / 2} \cdot(f(4))^{1 / 2} .
$$

Так как

$$
\left(\frac{\sqrt{2 \pi} \cdot 4^{4}}{48}\right)^{1 / 2} \cdot(f(4))^{1 / 2}=3.918722 \ldots,
$$

то

$$
|\bar{x}|_{2} \leqslant\left(\frac{n \rho}{4 e}\right)^{\rho / 2} \Delta^{\rho} \cdot 4 \rho^{9 / 4} \sqrt{n},
$$

и лемма доказана.

Следствие 2. Пусть выполнены условия леммы 2, а-максимум модулей элементов матрицы А. Предположим, что система линейных однородных уравнений (5) имеет решение с натуральными компонентами. Тогда эта система имеет решение $\bar{x}=\left(x_{1,0}, \ldots, x_{n, 0}\right)$ с натуральными компонентами $x_{j, 0}$, удовлетворяющее неравенству

$$
|\bar{x}|_{2} \leqslant 4(r+1)^{9 / 4} \sqrt{n} \cdot\left(\frac{n(r+1)}{4 e}\right)^{(r+1) / 2} \cdot k^{k(r+1) / 2} \cdot a^{k(r+1)} .
$$


Доказательство. Следует из неравенства Адамара и леммы 2.

Следующая наша цель состоит в том, чтобы вывести из оценки (20) оценку, в которой правая часть зависит лишь от чисел $n$ и $a$. Заметим, что так как $(x+1)(n-$ $x) \leqslant(n+1)^{2} / 4$ при любом $x, 0 \leqslant x \leqslant n$, то из неравенства $(20)$ легко вытекает

$$
|\bar{x}|_{2} \leqslant 4 n^{11 / 4} \cdot\left(\frac{n}{2 \sqrt{e}}\right)^{n} \cdot n^{(n+1)^{2} / 8} \cdot a^{(n+1)^{2} / 4} .
$$

В сформулированной выше теореме 1, к доказательству которой мы сейчас приступаем, дается асимптотически более точная верхняя оценка величины $|\bar{x}|_{2}$.

Ввиду оценки (20) для доказательства теоремы 1 достаточно доказать, что

$$
\left(\frac{n(r+1)}{4 e}\right)^{(r+1) / 2} \cdot k^{k(r+1) / 2} \leqslant\left(\frac{n+1}{\sqrt{8 e}}\right)^{(n+1) / 2} \cdot e^{(n+1)^{2} /(8 \ln (n+1))} \cdot\left(\frac{n+1}{2}\right)^{(n+1)^{2} / 8} .
$$

Пусть $R=r+1$ и $N=n+1$. Неравенство (21) будет верным, если при условии $k+R=N$ выполнено неравенство

$$
\left(\frac{N R}{4 e}\right)^{R / 2} \cdot k^{k R / 2} \leqslant\left(\frac{N}{\sqrt{8 e}}\right)^{N / 2} \cdot e^{N^{2} /(8 \ln N)} \cdot\left(\frac{N}{2}\right)^{N^{2} / 8} .
$$

Логарифмируя (22), получим, что надо доказать оценку

$$
R\left(\ln \frac{N R}{4 e}+k \ln k\right) \leqslant N\left(\ln \frac{N}{\sqrt{8 e}}+\frac{N}{4 \ln N}+\frac{N}{4} \ln \frac{N}{2}\right) .
$$

Напишем для целевой функции $f(R, k)=R\left(\ln \frac{N R}{4 e}+k \ln k\right)$ левой части формулы (23) функцию Лагранжа:

$$
F(R, k)=R\left(\ln \frac{N R}{4 e}+k \ln k\right)+\lambda(R+k-N),
$$

где $\lambda$ - множитель Лагранжа. Дифференцируя $F(R, k)$ по $R$ и $k$, получим, что экстремум функции $f(R, k)$ при условии $k+R=N$ должен удовлетворять равенству

$$
\ln R+1+k \ln k+\ln \frac{N}{4 e}=R \ln k+R .
$$

Покажем, что если числа $R$ и $k$ удовлетворяют $(24)$, то $R<\frac{N}{2}$. Допустим противное: $R \geqslant \frac{N}{2}$. Тогда $k<\frac{N}{2}$, следовательно, $k \ln k \leqslant R \ln k$. Равенство (24) в этом случае дает $\ln R+1+\ln \frac{N}{4 e} \geqslant R$, т.е. $\ln R+\ln \frac{N}{2} \geqslant R$. Для получения противоречия теперь достаточно показать, что неравенство $\ln R+\ln T \geqslant R$ при $R \geqslant T \geqslant 1$ невозможно. В справедливости последнего легко убедиться непосредственно, и этим наше утверждение доказано.

Положим $R=\frac{N}{2}(1-\theta), k=\frac{N}{2}(1+\theta)$, где $\theta$ - некоторое положительное число. Из равенства (24) следует, что $(k-R) \ln k<R$, т.е. $N \theta \ln \frac{N}{2}<\frac{N}{2}(1-\theta)$. Отсюда вытекает, что

$$
\theta<\frac{1 / 2}{1 / 2+\ln (N / 2)}=\frac{1}{2 \ln N+1-2 \ln 2}
$$

Покажем, что последняя величина в $(25)$ меньше, чем $\frac{1}{\ln N}$. Для этого надо показать, что $\ln N<2 \ln N+1-2 \ln 2$, т.е. что $\ln N>2 \ln 2-1$. Последнее верно, поскольку $N=n+1 \geqslant 2$.

Таким образом, мы показали, что $0<\theta<\frac{1}{\ln N}$. Оценим сверху функцию $f(R, k)$ в точке экстремума. Мы имеем

$$
f(R, k) \leqslant \frac{N}{2}(1-\theta)\left(\ln \frac{N}{4 e}+\ln \frac{N}{2}+\frac{N}{2}(1+\theta)\left(\ln \frac{N}{2}+\ln (1+\theta)\right)\right) .
$$


Так как $\ln (1+\theta)<\theta<\frac{1}{\ln N}$, то

$$
f(R, k) \leqslant \frac{N}{2}\left(\ln \frac{N}{4 e}+\ln \frac{N}{2}\right)+\frac{N^{2}}{4}\left(\ln \frac{N}{2}+\frac{1}{\ln N}\right),
$$

и формула (23) (а вместе с ней и теорема 1) доказана.

Используя теорему 1 , легко получить доказательство теоремы 2 . Пусть $h_{1}, \ldots, h_{r}$ - произвольный набор, на котором достигается минимум веса многочлена (2). Производя перемножение скобок в многочлене (2), получим

$$
\left(1-x^{h_{1}}\right) \ldots\left(1-x^{h_{r}}\right)=1+\sum_{m} \sum_{i_{1}<\ldots<i_{2 m}} x^{h_{i_{1}}+\ldots+h_{i_{2 m}}}-\sum_{n} \sum_{j_{1}<\ldots<j_{2 n+1}} x^{h_{j_{1}}+\ldots+h_{j_{2 n+1}}} .
$$

Будем говорить, что при степени $x^{C}$ многочлена $G(x)$ имеет место сокращение коэффициентов, если найдутся такие наборы индексов $\left(i_{1}, \ldots, i_{2 m}\right)$ и $\left(j_{1}, \ldots, j_{2 n+1}\right)$, что

$$
h_{i_{1}}+\ldots+h_{i_{2 m}}=h_{j_{1}}+\ldots+h_{j_{2 n+1}}=C .
$$

Будем говорить, что при степени $x^{C}$ многочлена $G(x)$ имеет место кратное сокращение коэффициентов, если равенства (26) имеют место для нескольких пар наборов индексов $\left(i_{1}, \ldots, i_{2 m}\right)$ и $\left(j_{1}, \ldots, j_{2 n+1}\right)$, в которых как левые части $\left(i_{1}, \ldots, i_{2 m}\right)$ так и правые части $\left(j_{1}, \ldots, j_{2 n+1}\right)$ попарно различны.

Выпишем для каждой степени $x^{C}$ многочлена $G(x)$ максимальную совокупность равенств вида (26), обеспечивающих кратное сокращение коэффициентов при данной степени. Объединяя все эти совокупности равенств, получим систему линейных однородных уравнений

$$
A \bar{h}=\overline{0}, \quad \bar{h}=\left(h_{1}, \ldots, h_{r}\right)^{t}
$$

относительно величин $h_{1}, \ldots, h_{r}$. Всякое натуральное решение $h_{1}^{*}, \ldots, h_{r}^{*}$ такой системы порождает многочлен

$$
G^{*}(x)=\left(1-x^{h_{1}^{*}}\right) \ldots\left(1-x^{h_{r}^{*}}\right),
$$

вес которого совпадает с весом многочлена $G(x)$.

По своему построению система $(27)$ имеет решение $\bar{h}=\left(h_{1}, \ldots, h_{r}\right)^{t}$ с натуральными компонентами $h_{j}$. Применяя теорему 1 , получим, что система $(27)$ имеет решение $\bar{h}_{0}=\left(h_{1,0}, \ldots, h_{r, 0}\right)^{t}$ с натуральными компонентами $h_{j, 0}$, удовлетворяющими неравенству (3), чем и завершается доказательство теоремы 2.

\section{Список литературы}

1. Касселс Дж.В.С., Введение в теорию диобантовых приближений, Изд. иностр. лит., Москва, 1961.

2. Корн Г., Корн Т., Справочник по математике, Наука, Москва, 1973.

3. Сазонов Д.М., Антенны и устройства СВЧ, Высшая Школа, Москва, 1988.

4. Схрейвер А., Теория линейного и целочисленного программирования, m.1, 2, Мир, Москва, 1991.

5. Фельдман Н.И., Седъмая проблема Гилъберта, Наука, Москва, 1982.

6. Borwein P., Erdélyi T., "On the zeros of polynomials with restricted coefficients", Illinois J. Math., 41 (1997), 667-675. 
7. Boyd D.W., "On a problem of Byrnes concerning polynomials with restricted coefficients", Math. Comput., 66:220 (1997), 1697-1703.

8. Boyd D.W., "On a problem of Byrnes concerning polynomials with restricted coefficients, II", Math. Comput., 71:239 (2001), 1205-1217.

9. Byrnes J.S., "Problems on polynomials with restricted coefficients arising from questions in antenna array theory", Recent Advances in Fourier Analysis and Its Applications, 1990, 677-678.

10. Byrnes J.S., Newman D.J., "Null steering employing polynomials with restricted coefficients", IEEE Trans. Antennas and Propagation, 36 (1988), 301-303.

11. Lagarias J.C., Lenstra H.W., Schnorr C.P., "Korkine - Zolotareff bases and successive minima of a lattice and its reciprocial lattice", Tech. Rept. MSRI 07718-86, Math. Sci. Res. Inst., Berkley, 1986, 122-146.

12. Mignotte M., "Sur les polynômes divisibles par $(X-1)^{n}$ ", Arithmetix, 1980, № 2, 28-29.

13. "Prometheus Inc.Polynomials with restricted coefficients and their applications", 88 0092, Tech. rep. Air Force Office of Scientific Research, 1990.

14. "Prometheus Inc. New approaches to beamforming, null steering and filtering", 900866 , Tech. rep. Air Force Office of Scientific Research, 1988.

15. Schnorr C.P., "A hierarchy of polynomial time lattice basis reduction algorithms", Theoretical Computer Science, 1987, № 53, 201-224.

16. Shoup V., "NTL: A Library for doing Number Theory // Dep. Comput. Sci., Wisconsin-Madison, http://www.shoup.net/". 